\title{
Una propuesta para la formación del educador musical en tiempos de cambio
}

\author{
Elsa Cristina Navarrete Ochoa \\ Universidad Autónoma de Querétaro
}

\section{Introducción}

Hacia la segunda década del nuevo siglo se observan importantes transformaciones en el contexto mundial, propiciadas por una multiplicidad de factores que impactan sobre los grupos sociales y la manera en que se construyen nuevos paradigmas culturales. La ruptura de fronteras territoriales permite el encuentro multicultural en amplio sentido. Los intercambios que se movilizan impactan en todos los niveles: económico, de producción, ecológico, científico, tecnológico, artístico, etc. Las formas de comunicación actual han hecho posible una revolución que pone a los jóvenes profesionistas ante nuevas posibilidades con las que pueden redefinir sus acciones para la vida adulta, y resolver las complejidades del futuro que heredan con iniciativas novedosas. Se han apropiado de causas como el rescate de las culturas indígenas, la conservación del ambiente, la construcción de la identidad ante contextos de multiculturalidad, en fin, respuestas innovadoras provenientes de encuentros interdisciplinarios entre diferentes campos de estudio, lo que trae consigo nuevos retos a los formadores en las instituciones de educación superior.

En el presente artículo se expondrán brevemente algunas consideraciones que han llevado a académicas del nivel superior a influir en las prácticas y programas de formación de educadores musicales, y propuestas consecuentes con la intención de adecuar los contenidos y las formas a los requerimientos del escenario actual, y aproximar a las y los estudiantes a contextos vinculados con la realidad de las tareas de su profesión.

\section{La enseñanza de la música}

Los conservatorios de música, fundados desde el siglo XVI en Europa, así como las escuelas de música de nivel superior influenciadas por los primeros, se han tardado en modificar tanto sus programas de estudio como sus prácticas educativas. Muchos de los principios pedagógicos se han repetido tradicionalmente desde entonces sin experimentar cambios importantes en las relaciones entre los estudiantes y sus maestros, o en la forma de comprender el hecho musical y la tarea del músico profesional:

A lo largo del siglo XIX, en diferentes países europeos, y en forma correlativa en algunas de las grandes capitales del nuevo mundo, se ponen en vigencia las estructuras musicales, programas, repertorios $\mathrm{y}$ textos que irradian los principales centros, constituyéndose así en el punto de referencia básico respecto de la calidad y el alcance de la enseñanza musical que imparten las instituciones educativas (Hemsy, 1999, p. 2).

Para los países Latinoamericanos la realidad no es muy diferente ya que han sido repetidores, antes que innovadores, de estos modelos extendidos desde Europa a pesar de los sí significativos avances para la educación inicial. Una de las más sobresalientes figuras precursoras para la educación musical 
en Latinoamérica, Violeta Hemsy de Gainza ha llamado al siglo XX como el siglo de la iniciación musical, puesto que desde las primeras décadas del siglo pasado, los promotores de la pedagogía musical han cuestionado estas prácticas tradicionalistas y como resultado surgieron interesantes métodos para la educación e iniciación musical: en Europa Dalcroze, Orff, Kodaly, posteriormente para Europa y Asia Willems y Suzuky, en México César Tort, en Argentina Violeta Hemsy, en Canadá M. Schafer, entre otros.

Pese a esto, la educación musical en el nivel superior no se ha visto todavía reformada:

la propuesta educativa del conservatorio, la máxima institución de formación musical durante los siglos XIX y XX en nuestras latitudes, curiosamente, no se ha modificado a lo largo de una época signada por transformaciones realmente extraordinarias que han incidido en la manera de ser, de pensar y de comunicarse con la gente, en el ritmo de la vida cotidiana, en el uso y la distribución del tiempo libre, el estudio y el trabajo, en las apetencias musicales de niños y jóvenes, etc., hechos éstos que determinan cambios esenciales en la función del arte en la sociedad (Hemsy, 1999. p.7).

Entre los diagnósticos hechos hacia la necesidad de transformar los programas para la formación de los educadores musicales, se detecta la importancia de promover un cambio de perspectiva que significa un giro de 180 grados. Nuevas concepciones son necesarias ante la realidad de los cambios en el rol del arte y de la música en las sociedades actuales. Por citar algunas, Andrés Samper propone pensar en nuevas epistemologías y aproximaciones didácticas para abordar la interculturalidad. Lucy Green y María Teresa Díaz, entre otras, señalan la relevancia de reflexionar acerca de las prácticas musicales y sus repercusiones en temas de género. Lines y Schafer, enfatizan el valor de la de la educación de la sensorialidad y la improvisación. Hemsy de Gainza recomienda puntualmente considerar para nuevos programas tres grandes áreas: aspectos técnico-musicales, aspectos culturales y aspectos psicopedagógicos en una "propuesta curricular organizada en torno a ejes que remiten a los elementos o estructuras de la música” (Hemsy, 1999, p. 11).

Por otro lado, desde la pedagogía general, los diseñadores curriculares han identificado en el aprendizaje colaborativo estrategias que han resultado trascendentales en la práctica educativa por sus aportaciones, con la consigna de conseguir vincular los aprendizajes escolares con la vida cotidiana. De este modo y en una síntesis que integra las preocupaciones de los educadores musicales y las de las tendencias de orden didáctico-pedagógico, se han adoptado estrategias para el aprendizaje basado en problemas (ABP) y proyectos (ABP) para promover los procesos de aprendizaje con estudiantes de nivel superior del programa de la Licenciatura en Música, línea terminal en educación, de la Facultad de Bellas Artes (FBA) de la Universidad Autónoma de Querétaro (UAQ).

Algunos autores consideran que aprender a manejar proyectos y colaborar con ellos, entendiéndolos como una forma idónea de acción colectiva, es uno de los aprendizajes más significativos que puede logar una persona, pues incide tanto en su faculta- 
miento o construcción de una identidad personal sólida como en su preparación para el trabajo colectivo y la ciudadanía (Díaz, 2006, p. 30).

De tal modo resulta que aprender y hacer son acciones inseparables y por lo tanto los/las alumnos requieren aprender en contextos pertinentes. La preparación de actividades basadas en las estrategias de aprendizaje orientado a proyectos y de resolución de problemas, facilitan la puesta en escena de acciones movilizadoras durante el proceso de aprendizaje, en que los contenidos disciplinares y la práctica son tomados por el sujeto cognoscente en una interacción permanente, en la que éste aprende como resultado de su actividad. Esta participación además compuesta por el sujeto situado en grupos de colaboración potencializa sus capacidades en tanto que pone a prueba sus aprendizajes en el diálogo con sus pares y a la vez le permite modelar sus actitudes, con lo que se le aproxima al alcance de su desarrollo integral. Es decir se le pone en situaciones que le permitan desenvolver y consolidar el desarrollo de sus habilidades, conocimientos y actitudes. Los acuerdos de la Academia de este programa educativo para educadores musicales en el año 2014, consideran como parte de la formación de los estudiantes asignaturas como: Diseño curricular, Sociología de la educación, los métodos de la pedagogía musical del siglo XX y modelos para el siglo XXI, la gestión cultural y de proyectos entre otros, con un enfoque basado en las teorías del socioconstructivismo mediante el diseño de situaciones contextualizadas. La forma de trabajo que se propuso por medio de estas estrategias permitió que desde el inicio se propiciaran formas de relación entre los participantes: se tomaron acuerdos para delimitar el proyecto $\mathrm{y}$ conducirlo, se delegaron funciones y se llevaron a cabo acciones de estudio e inves- tigación que condujeran a la resolución del proyecto o el diseño y análisis de soluciones. El punto de partida fue la búsqueda de situaciones educativas en entornos específicos para educación musical. Las estrategias para la evaluación fueron también congruentes con la perspectiva constructivista de manera que se observara el desempeño del alumno en su integralidad $y$ durante los diferentes momentos del proceso educativo.

En seguida se presenta la descripción de actividades con estas características aplicadas a alumnas/os de cuarto a sexto semestres de dicho programa educativo. El objetivo general en los siguientes ejemplos estuvo relacionado con promover la disposición de experiencias y prácticas necesarias que permitieran a los y las estudiantes: conocer, indagar, analizar e identificar, datos pertinentes para el diseño de situaciones contextualizadas para educación musical.

\section{Proyecto: Banco de situaciones para la} educación musical (Enero-junio de 2014) El Proyecto "Banco de situaciones para la educación musical” se propuso para los contenidos de diseño curricular. Se consideró como un antecedente central en el desarrollo de los siguientes proyectos que se suceden en consecuencia. Se pretende que las y los estudiantes se apropien del conocimiento teórico mediante la resolución de propuestas prácticas. El logro de este proyecto llevó a realizar las siguientes acciones organizadas en dos momentos, uno teórico y otro práctico a veces sucedidos simultáneamente:

\section{Investigación bibliográfica y análisis de los datos:}

a) Estudio de los antecedentes: Pedagogía por Objetivos (PPO), sus características y aportaciones.

b) Enfoque constructivista. 
c) Estrategias para el aprendizaje colaborativo.

d) Evaluación holística.

\section{Intervención práctica:}

a) Como resultado de la etapa anterior se planteó como problema el siguiente: “Aprendices de laudero”. El propósito fue conducir el ejercicio para la detección de elementos para el diseño curricular. Contar con una situación contextualizada permitió tomar decisiones objetivas y apegadas a un contexto real.

b) Evaluación holística. Estrategias para la evaluación diagnóstica, formativa y sumativa, que se realizaron durante la aplicación del proyecto con los niños de la Comunidad Amolitos, Cadereyta, Querétaro.

\section{Proyecto: Aprendices de laudero.}

Construcción de cotidiáfonos para niños de la comunidad de Amolitos Cadereyta, Querétaro. (Enero-junio de 2014)

Este proyecto es la última fase del proyecto anterior que concluyó con el diseño para la intervención que se describirá en este apartado. Se planteó como una situación concreta y contextualizada que permitiera a las y los alumnos llevar a cabo la realización de un plan de trabajo desde la Pedagogía por Objetivos. Posteriormente y con el propósito de reflexionar sobre el enfoque basado en competencias como requerimiento del sistema educativo nacional, se condujo hacia su conocimiento y análisis crítico. De esta manera se motivó a que los y las estudiantes tomaran conciencia de las exigencias de la tarea educativa desde las actividades iniciales que se requieren en el diseño curricular. El planteamiento que se logró fue el siguiente:
Aprendices de laudero: Propósito: exploración y definición de las cualidades del sonido.

\section{Actividades etapa 1:}

a. Identificación y conceptualización. Detección de actividades que llevaran a cumplir con el objetivo.

b. Entrevista en el taller de laudería ViolinArt al laudero queretano Armando Plancarte. Se grabó video que se llevó como actividad de enfoque a los niños/as de la comunidad de Amolitos.

c. Visualización de las vibraciones del sonido. Experimento con un globo, una lata, luz, un espejo, muchos niños curiosos y tres estudiantes de educación musical muy entusiastas.

d. Actividades de experimentación para conocer las cualidades del sonido.

e. Construcción de cotidiáfonos.

f. Ensamble vocal e instrumental con los instrumentos construidos por los niños

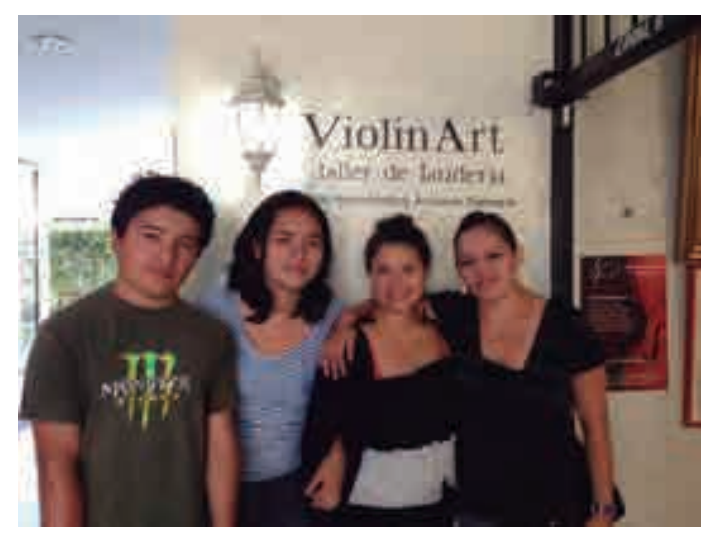

Estudiantes de cuarto semestre de la Licenciatura en Música, línea terminal en Educación Musical de la FBA de la UAQ, en el taller de laudería ViolinArt, el día de la entrevista al laudero Armando Plancarte.

Estudiantes del cuarto semestre de la Licenciatura en Música, línea terminal en 


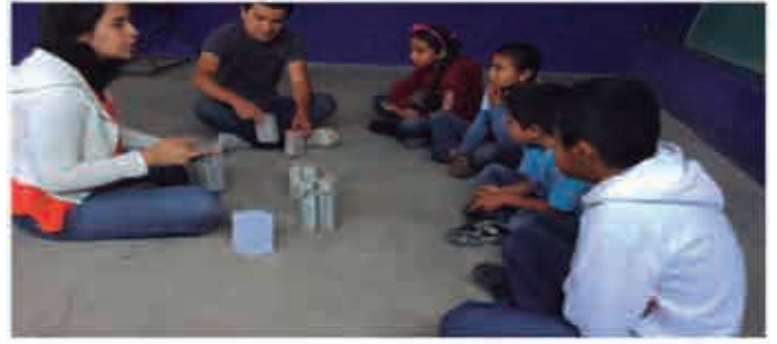

Educación Musical de la FBA de la UAQ, en el desarrollo de las actividades del proyecto “Aprendices de laudero” con los niños de la comunidad de Amolitos Cadereyta, Querétaro.

Actividades etapa 2: Propósito: Conocer y reflexionar acerca del enfoque basado en competencias:

a) Análisis y reflexión de las perspectivas hacia el enfoque de competencias.

b) Discusión y recomendaciones para abordar

el diseño curricular desde el enfoque basado en competencias.

c) Identificación de contenidos disciplinares para la educación musical.

\section{Intervención práctica:}

a) Identificación de situaciones contextualizadas para la definición de competencias en educación musical en los diferentes niveles educativos de la educación básica (banco de situaciones).

Al término del semestre el proyecto fue presentado en exposición de una mesa temática en el IV Encuentro de Educadores Musicales en Querétaro. Como iniciativa surgida del proyecto se abrió un espacio en línea para continuar con el banco de situaciones y promoverlo con otros estudiantes de otros semestres y de otras instituciones.

\section{Proyecto para la estimulación temprana con educación musical. (Agosto de 2013 a mayo de 2014)}

Este proyecto se desarrolló en seguimiento de los estudios semestrales correspondientes al diseño curricular en atención a las propuestas de los estudiantes y en tanto que les permitiría desarrollar sus habilidades como investigadores para la educación musical. En construcción colectiva del proyecto se propusieron los siguientes pasos:

a. Indagar y conocer las características que supone la etapa de desarrollo de los cero a los tres años de edad desde el desarrollo psicomotriz, neuronal, intelectual y afectivo.

b. Se hizo una investigación local para localizar a las instituciones que ofrecían estimulación temprana en términos cuantitativos. (No alcanzó a ser cualitativo por el hermetismo que se encontró en dichas instituciones).

c. Se establecieron paralelismos entre los objetivos de la estimulación temprana con los propios de la educación musical, y se diferenciaron los momentos de desarrollo por edades en estos niños.

d. Se identificaron fuentes bibliográficas tanto físicas como virtuales.

e. Se observó a profesionales de la educación involucrados con la etapa de interés.

f. Se observó a profesionales que hacen educación musical en esta etapa.

g. Se diseñó un proyecto, con la posibilidad de dar base a otros posteriores, que abarca las diferentes etapas de los cero a los tres años de edad.

h. Algunas actividades fueron aplicadas durante la Semana Cultural de un Colegio local.

i. Se participó dentro del programa de la FBA "Miércoles de Concierto", como parte de las actividades del "IV encuentro de Educadores Musicales en Querétaro”. Se presentó como un taller de actividades musicales para la estimulación temprana, abierto al público general en donde los estudiantes además de demostrar la aplicación de las actividades que prepararon, pudieron justificar su pertinencia 
en el desarrollo infantil.

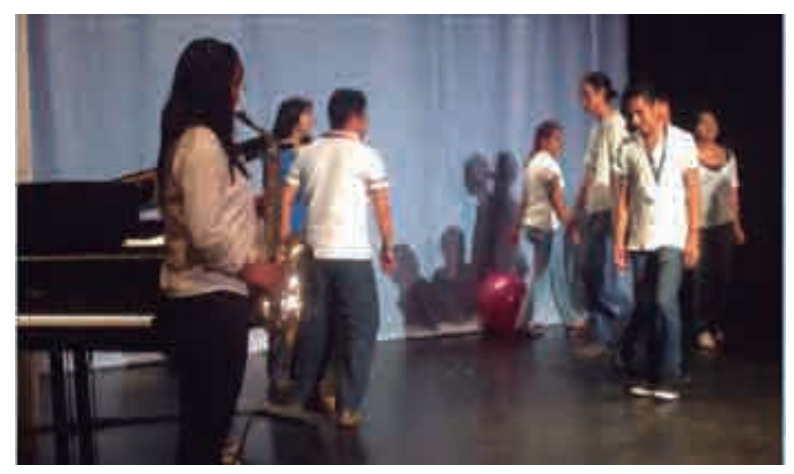

Exploración de las cualidades del sonido: duración

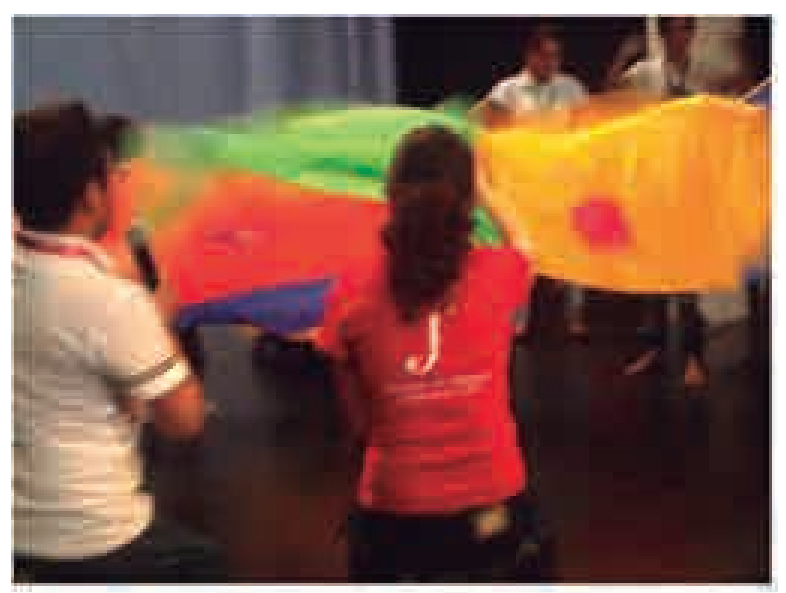

Sensibilización sensorial

Estudiantes de sexto semestre de la Licenciatura en Educación Musical de la FBA de la UAQ, durante el IV Encuentro de educadores Musicales en Querétaro, en el taller de actividades Musicales para Estimulación Temprana. Mayo de 2014.
Lo anterior dio origen a nuevas repeticiones del taller en demanda de la necesidad en Jardines de Niños y Estancias Infantiles en solicitud de las maestras encargadas. Como parte de esta edición de talleres se realizó la grabación de los cantos y juegos musicales de estos talleres y se pudo ofrecer el disco como apoyo didáctico.

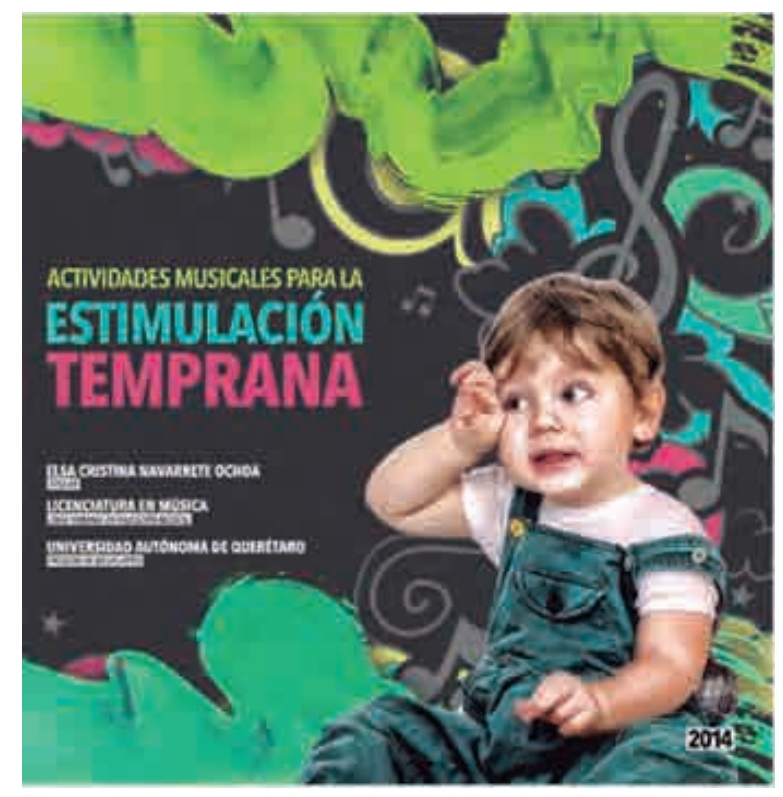

Carátula frontal del CD grabado en noviembre de 2014 por estudiantes de la Licenciatura en Música, línea terminal en Educación Musical de la FBA de la UAQ, "Cantos y juegos musicales para estimulación temprana”. 
IV Encuentro de Educadores Musicales en Querétaro. (Mayo de 2014)

El Encuentro de Educadores Musicales en Querétaro es un espacio que se ideó para incluir actividades complementarias al programa de formación. Se origina con una doble intencionalidad:

Por un lado, es una oportunidad de implementar actividades que fortalezcan el programa educativo por medio de actividades en la que los y las alumnas participan en mesas temáticas, y se enfrentan a una audiencia ante la que deberán:

a. Estructurar argumentativamente su exposición, y aplicar de manera integral los contenidos de estudio.

b. Argumentar, explicar, respaldar y defender sus argumentaciones en la construcción de su conocimiento.

c. Conciliar con sus compañeros de grupo, bajo la óptica del grupo colaborativo, en que el trabajo individual exige un compromiso solidario ante una meta común.

d. Superar las relaciones jerárquicas y promover el diálogo.

e. Definir expectativas, y actuar disciplinadamente para afrontar las exigencias que impone presentarse ante una audiencia en un marco de intercambios académicos.

f. Demostrar su esfuerzo y compromiso.

Por el otro lado, se ha dicho que se consideró de doble intención ya que además se pretende facilitar el encuentro con maestros en educación básica y estudiantes, con el propósito central de sensibilizarlos hacia el reconocimiento de la educación musical como parte integradora en sus acciones educativas (en educación básica general), y sobre todo al reconocimiento de los profesionistas en esta área para promover la comprensión y valoración de la necesidad de contar para las tareas de educación artística y musical con los educadores especializados.

\section{Conclusiones}

Es necesario observar, estudiar, comprender y analizar críticamente los cambios y exigencias del contexto contemporáneo a fin de actualizar los programas y las prácticas educativas y que estas sean congruentes con los avances y las necesidades sociales. En consonancia con lo que sugiere Hemsy de Gainza (1999): no se debe permanecer al margen de los cambios generalizados que se registran a nivel de:

- las características de personalidad de las nuevas generaciones; sus estructuras mentales, su necesidad de expresión, sus formas de comunicación, sus expectativas y aficiones musicales; - los nuevos modelos institucionales para la formación musical profesional; las nuevas carreras musicales y sus respectivas salidas laborales; el campo audiovisual y de la comunicación, la integración artística, etc.;

- la importancia creciente de la música popular no comercial que, en sus múltiples formas, ha atenuado las fronteras que tradicionalmente separaban los diferentes estilos y géneros musicales;

- las nuevas problemáticas sociales y su incidencia en la educación musical; los avances tecnológicos el multiculturalismo, la ecología, el poder de los medios y su influencia social;

- la necesidad de una pedagogía actualizada de la música que permita responder a las necesidades musicales individuales y sociales de la población.

En ese sentido se sugiere la aplicación de la educación musical a la resolución de problemas sociales que nos permitan educar para la sana convivencia. Motivar la reflexión hacia los acontecimientos con los que culturalmente convivimos cotidianamente, atentos a sus repercusiones sobre la conciencia social y proponer formas de intervención hacia la construcción de entornos preparados para estilos de vida de mayor equilibrio entre lo humano y la vida civil, en lo individual y lo colectivo. 


\section{Bibliografía}

Arias, M. (2007). Música y neurología. Santiago de Compostela: Neurología 2007

Cortinas del Riego, M. (1983). Curso euritmia-Dalcroze. México. D.F. México: UNAM.

Cortinas del Riego, M. (1984). Curso euritmia-Dalcroze. México. D.F. México: UNAM.

Dalcroze, J. (1923). 50 Etudes miniatures de métrique et rythmique. París: Éditions Maurice Senart.

Delval, J. (2001). Hoy todos son constructivistas. Mérida. Venezuela: Educere.

Díaz Barriga. F. (2006). Enseñanza situada: Vínculo entre la escuela y la vida. México. D.F.: McGraw-Hill/Interamericana.

Díaz-Barriga, A. (2011). Competencias en educación. Corrientes de pensamiento e implicaciones para el currículo y el trabajo en el aula. Revista Iberoamericana de educación Superior. México: ISSUE-UNAM/Universia.

Findlay, E. (1971). Rhytm and movement: Applications of Dalcroze eurhytmics. Miami: Summy-Bitchard Inc.

Frade Rubio, L. (2011). Elaboración de rúbricas: Metacognición y aprendizaje. México. D. F.: Inteligencia educativa.

Hemsy de Gainza, V. (1986). La improvisación musical. Buenos Aires: Ed. Ricordi.

Hemsy de Gainza, V. (1987). La iniciación musical del niño. Buenos Aires: Ed. Ricordi.

Hemsy de Gainza, V. (1992). Para divertirnos cantando. Cancionero. Buenos Aires: Ed. Ricordi.

Hemsy de Gainza, V. (1999). La educación musical superior en Latinoamérica y Europa durante el siglo XX. Realidad y perspectivas. Doce notas, 3.

Hemsy de Gainza, V. (2003). Pedagogía musical: Dos décadas de pensamiento y acción educativa. Buenos Aires: Lumen,

Herrera, R. (1999). La intercomunicación en el aula. Morelia: IMCED.

Jonnaert, P., Barrette, J., Masciotra , D., \& Yaya, M. (2006). Revisión de la competencia como organizadora de los programas de formación: hacia un desempeño competente. Observatorio de Reformas Educativas. Quebec: ORÉ/UQAM.

Matas, S. (2006). Estimulación temprana de 0 a 36 meses. Argentina: Lumen-Hvmanitas.

Pascual Mejía, P. (2002). Didáctica de la música para primaria. Madrid: Pearson Educación

Perrenaud, P. (2006). Construir competencia desde la escuela. Santiago: Ediciones Noreste.

Samper Arbeláez, A; (2011). Educación musical a nivel superior e interculturalidad en el siglo XXI: nuevas epistemologías, nuevas aproximaciones didácticas. El Artista, () 297-316.

Recuperado de http://www.redalyc.org/articulo.oa?id=87420931020

Sanuy, Montse y Conchita. (1988). Al son que tocan, bailo. Bogotá: Cincel Kapeluz.

Soria-Urios G, Duque P, García-Moreno J.M. (2011) Música y cerebro: fundamentos neurocientíficos y trastornos musicales. Rev. Neurol. 2011; 52. (pp. 45-55). Recuperado de internet en 2011. http://www.neurologia.comwww.neurologia.com

Soria-Urios G, Duque P, García-Moreno J.M. (2011) Música y cerebro (II): evidencias cerebrales del entrenamiento musical. Rev. Neurol. 2011; 53. (pp. 739-46). Recuperado de internet en 2011. http://www.neurologia.com www.neurologia.com 


\section{INFORMES DE INVESTIGACIÓN}

Steinitz, T. (1988). Teaching music in rhytmic lessons: Theory and practice of the Dalcroze method. Tel-Aviv: Or-Tav, Music publications.

Talero-Gutiérrez, C. (2004) Percepción musical y funciones cognitivas: ¿Existe el efecto Mozart? Recuperado de internet en 2004. http://www.neurologia.com

Tort, C. (s/f). El niño descubre la música. Primera parte: Estimulación musical temprana. México, D. F.: UNAM.

Willens, E. (1964). El ritmo musical. Buenos Aires: Editorial Universitaria de Buenos Aires. Willens, E. (1994). El valor humano de la educación Musical. Barcelona: Paidós.

Yelin, J. (1990). Movement that fits: Dalcroze euthytmics and the Suzuki method. Secaucus. New Jersey: Summy-Bitchard Inc.

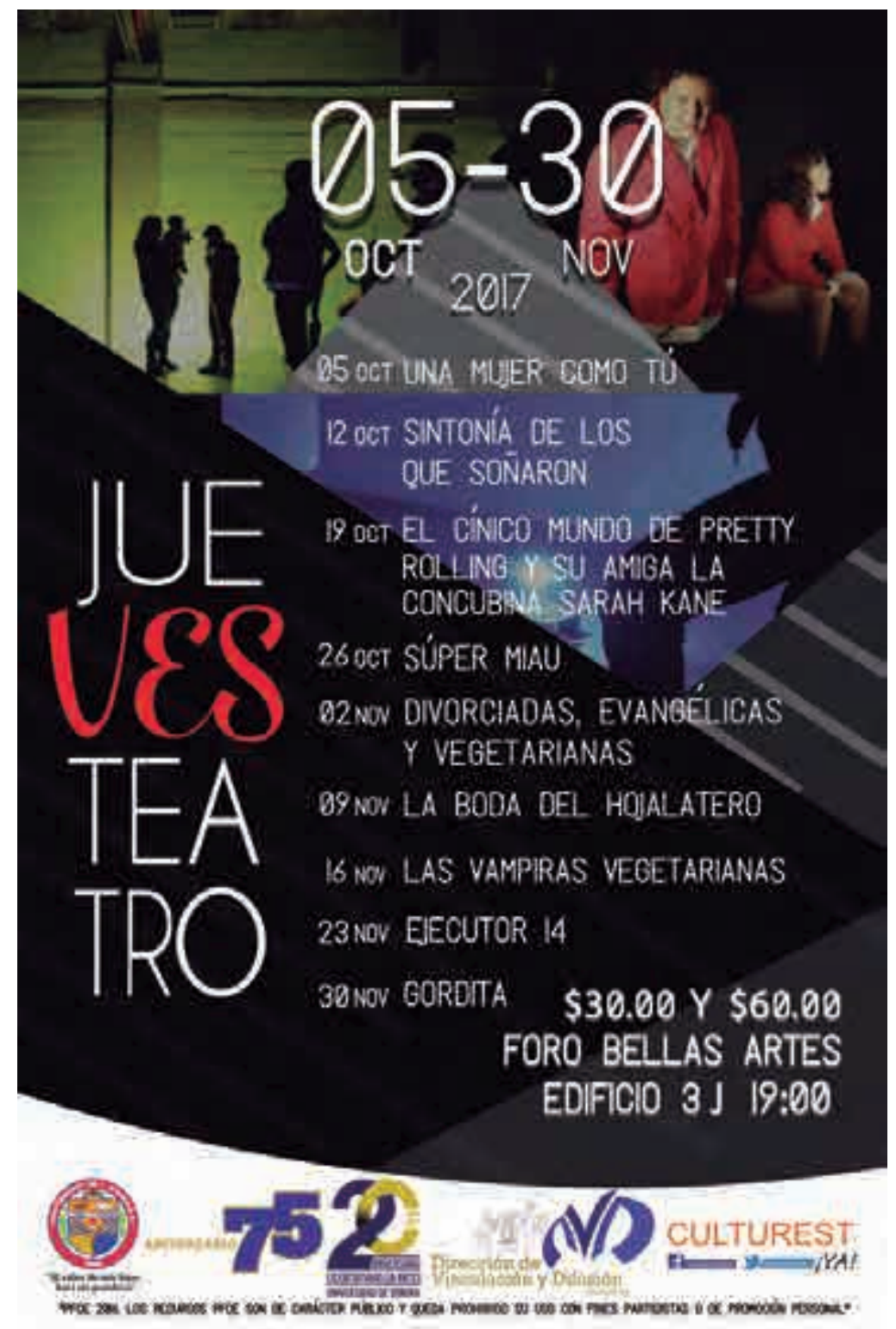

Jueves Teatro 